Anal ysi s of 8 hydr oxy-2' - deoxyguanosi ne i n human ur i ne usi ng hydrophi I i c i nt er act i on chr omat ogr aphy wi th tandem mass spect r omet $r y$

\begin{tabular}{|c|c|}
\hline 著者 & $\begin{array}{l}\text { Hosozumi Chi emi, Tor i ba Aki ra, Chuesaard } \\
\text { Thanyar at, Kaneda Takayuki, Tang N ng, } \\
\text { Hayakawa Kazui chi }\end{array}$ \\
\hline $\begin{array}{l}\text { jour nal or } \\
\text { publ i cat } i \text { on title }\end{array}$ & $\begin{array}{l}\text { Jour nal of Chr omat ogr aphy B: Anal yt i cal } \\
\text { Technol ogi es in the Bi onedi cal and Li fe } \\
\text { Sci ences }\end{array}$ \\
\hline vol une & $893-894$ \\
\hline page $r$ ange & 173176 \\
\hline year & 2012-04 15 \\
\hline URL & ht t p: //hdl . handl e. net /2297/31391 \\
\hline
\end{tabular}




\section{Analysis of 8-hydroxy-2'-deoxyguanosine in human urine using hydrophilic interaction chromatography with tandem mass spectrometry}

Chiemi Hosozumi ${ }^{\text {a }}$, Akira Toriba ${ }^{\mathrm{a}, *}$, Thanyarat Chuesaard ${ }^{\mathrm{a}}$, Takayuki Kameda ${ }^{\mathrm{a}}$, Ning Tang $^{\mathrm{b}}$, Kazuichi Hayakawa ${ }^{\mathrm{a}}$

${ }^{a}$ Institute of Medical, Pharmaceutical and Health Sciences, Kanazawa University, Kakuma-machi, Kanazawa 920-1192, Japan

${ }^{\mathrm{b}}$ Department of Public Health, Hyogo College of Medicine, 1-1 Mukogawa-cho, Nishinomiya 663-8501, Japan

*To whom correspondence should be addressed.

Tel.: +81 76234 4457; fax: +81 762344456 .

E-mail address: toriba@p.kanazawa-u.ac.jp 


\section{Abstract}

Urinary 8-hydroxy-2'-deoxyguanosine $(8-\mathrm{OHdG})$ is a widely used noninvasive biomarker of oxidative stress. A selective, sensitive and rapid method for determining 8-OHdG in human urine was developed using hydrophilic interaction chromatographytandem mass spectrometry (HILIC-MS/MS) with electrospray ionization. 8-OHdG and isotopically labeled 8-OHdG (internal standard) were separated on a HILIC column with a mobile phase of $10 \mathrm{mM}$ ammonium acetate : acetonitrile $(1: 9, \mathrm{v} / \mathrm{v})$ within 10 min and detected by using a positive electrospray ionization interface under the selected reaction monitoring mode. The detection limits of $8-\mathrm{OHdG}$ (corresponding to a signal-to-noise ratio of 3) for the HILIC-MS/MS system and the conventional method using a reversed-phase column with MS/MS were 1.0 and 26.0 fmol/injection, respectively. The proposed method makes it possible to monitor the basal level of urinary $8-\mathrm{OHdG}$ from non-exposed healthy subjects and can be used for large-scale human studies.

Keywords: Hydrophilic interaction chromatography, Tandem mass spectrometry, 8-Hydroxy-2'-deoxyguanosine, Urine, Oxidative stress 


\section{Introduction}

Oxidative stress in an organism arises from excessive generation of reactive oxygen species (ROS) such as superoxide radicals, hydrogen peroxide and hydroxyl radicals or from depletion of antioxidants [1]. The production of ROS can be induced by both endogenous and exogenous factors [2]. While endogenous factors include physiological processes, exogenous factors include environmental sources such as smoking, diet and pollution [3]. ROS may cause oxidative damage to nucleic acids, proteins, and lipids [2]. In particular, oxidative damage to DNA has been associated with numerous pathological conditions, having both genetic and epigenetic consequences [4-6]. To understand how ROS affect normal and pathological processes, an indicator to assess oxidative stress in vivo is required.

The oxidized product of DNA, 8-hydroxy-2'-deoxyguanosine (8-OHdG), is the most frequently measured biomarker of the oxidative stress $[7,8]$. The $8-\mathrm{OHdG}$ has been analyzed in various kinds of samples, such as urine, serum, peripheral blood leukocyte, and organ tissue [9]. Measurements of $8-\mathrm{OHdG}$ in urine samples are especially well-suited to large-scale human studies and clinical applications because they are noninvasive $[10,11]$. Urinary $8-\mathrm{OHdG}$ has been analyzed by several methods, such as enzyme-linked immunosorbent assay (ELISA) [12, 13], high-performance liquid 
chromatography with electrochemical detection (HPLC-ECD) [14], gas chromatography with mass spectrometry (GC/MS) $[15,16]$, and liquid chromatography with tandem mass spectrometry (LC/MS/MS) [17-26]. The ELISA method suffers from the problem of non-selectivity because the antibody may cross-react with other substances present in urine [12, 27, 28]. HPLC-ECD has often been used [29], but it suffers from possible interference from the biological matrix, incompatibility of a stable isotope labeled internal standard $[16,21]$. For GC/MS analysis, 8-OHdG must be purified by HPLC and derivatized before analysis $[15,16,28]$.

LC-MS/MS has been increasingly applied to detect urinary 8-OHdG. LC-MS/MS, when combined with the isotope dilution technique, is highly selective, sensitive, and accurate, and does not require derivatization [28]. In most of previous LC-MS/MS studies, reversed-phase columns have been used to separate 8-OHdG [17-26]. As a polar compound, 8-OHdG is hardly retained on a reversed-phase column, even though aqueous mobile phases are used. Such poor retention of $8-\mathrm{OHdG}$ and its insufficient separation from polar components in the matrix may lead to matrix effects, which can increase or decrease the $8-\mathrm{OHdG}$ MS signal [30]. In addition, aqueous mobile phases that are used to retain polar compounds on reversed-phase columns are not suited for electrospray ionization (ESI) conditions [30]. Indeed, urinary 8-OHdG levels have even 
been below the detection limit of LC-MS/MS method (7.5 fmol/injection, $\mathrm{S} / \mathrm{N}=3$ ) [27, 31]. Therefore, a more sensitive LC-MS/MS method is required to measure basal levels of urinary $8-\mathrm{OHdG}$ in non-exposed healthy subjects.

In recent years, hydrophilic interaction chromatography (HILIC) has been increasingly used in LC-MS/MS for analyzing polar compounds such as metabolites in biological samples. Under HILIC conditions, the analyte interacts with a hydrophilic stationary phase and is eluted with a high concentration of organic solvent (typically acetonitrile with a small percentage of water/buffer). The highly organic mobile phase can result in increased sensitivity with ESI-MS detection [30, 32].

In this study, we developed an improved LC-MS/MS method for measuring urinary 8-OHdG using a HILIC column. We found that the HILIC column provided much greater sensitivity than a conventional reversed-phase column with the same MS/MS detection conditions.

\section{Experimental}

\subsection{Materials}

The $8-\mathrm{OHdG}$ and $\left[{ }^{15} \mathrm{~N}_{5}\right] 8-\mathrm{OHdG}$ were purchased from Sigma (MO, USA) and Cambridge Isotope Laboratories (MA, USA), respectively. HPLC grade acetonitrile was 
obtained from Kanto Chemical (Tokyo, Japan), and water from a Milli-Q water purification system (Millipore, Bedford, MA, USA). All other chemicals and solvents used were of an analytical grade.

\subsection{Samples preparation}

Urinary samples were pretreated as described previously with a slight modification $[22,28]$. After centrifugation at $2150 \times \mathrm{g}$ for $10 \mathrm{~min}$, a $100 \mu \mathrm{L}$ aliquot of each supernatant was diluted with $900 \mu \mathrm{L}$ of water and spiked with 10 pmol of the stable isotope labeled internal standard (IS), $\left[{ }^{15} \mathrm{~N}_{5}\right] 8-\mathrm{OHdG}$. The diluted sample was subjected to solid-phase extraction using Oasis HLB cartridge (3 cc, 60 mg; Waters, Milford, MA, USA) that had been primed with $1 \mathrm{~mL}$ of methanol and $1 \mathrm{~mL}$ of water. After sample loading, the cartridge was sequentially washed with $1 \mathrm{~mL}$ of water. The $8-\mathrm{OHdG}$ was eluted from the cartridge with $500 \mu \mathrm{L}$ of water : acetonitrile $(1: 1, \mathrm{v} / \mathrm{v})$, and evaporated to dryness using a centrifugal vacuum evaporator. The residue was redissolved in 100 $\mu \mathrm{L}$ of water : acetonitrile $(1: 9, \mathrm{v} / \mathrm{v})$, and an aliquot of $20 \mu \mathrm{L}$ was injected into the $\mathrm{LC}-$ MS/MS system.

2.3. 8-OHdG analysis by HILIC-MS/MS 
2.3.1. Apparatus and chromatographic conditions

The Agilent 1100 series LC system consists of a G1379A degasser, a G1312A binary pump, a G1367A autosampler, and a G1316A column oven (all from Agilent Technologies, Palo Alto, CA, USA). The chromatographic separation of 8-OHdG in the pretreated urine samples was performed on a COSMOSIL HILIC $(150 \mathrm{~mm} \times 2.0 \mathrm{~mm}$ i.d., $5 \mu \mathrm{m}$, Nacalai Tesque, Kyoto, Japan), kept at the temperature of $40^{\circ} \mathrm{C}$. The column was eluted isocratically with $10 \mathrm{mM}$ ammonium acetate : acetonitrile $(1: 9, \mathrm{v} / \mathrm{v})$ at a flow rate of $0.4 \mathrm{~mL} / \mathrm{min}$. The retention time of the analyte was optimized by varying the mobile phase acetonitrile content between $70 \%$ and $95 \%$ with $10 \mathrm{mM}$ ammonium acetate and by varying the aqueous buffer concentration between 10 and $50 \mathrm{mM}$ with $90 \%$ acetonitrile. The retention factor $(k)$ of $8-\mathrm{OHdG}$ was defined as $k=\left(\mathrm{t}_{\mathrm{R}}-\mathrm{t}_{0}\right) / \mathrm{t}_{0}$, where $t_{R}$ and $t_{0}$ are the retention times of the analyte and the hold-up time, respectively. Sample volumes of $20 \mu \mathrm{L}$ were injected for each analysis. The mass spectrometric analyses were performed using an API 4000 Q-Trap tandem mass spectrometer (Applied Biosystems, CA, USA) equipped with an electrospray ionization (ESI) and operated in a positive ion mode. Sensitivity of the selective reaction monitoring (SRM) was optimized by testing with an infusion of $8-\mathrm{OHdG}$ under the mobile phase condition. The mass spectrometer was operated under SRM mode of the transitions at $m / z 284.3 \rightarrow$ 
167.9 for $8-\mathrm{OHdG}$ and at $\mathrm{m} / \mathrm{z} 289.1 \rightarrow 173.0$ for $\left[{ }^{15} \mathrm{~N}_{5}\right] 8-\mathrm{OHdG}(\mathrm{IS})$ with dwell times of $1000 \mathrm{~ms}$. The spray voltage was maintained at $5.5 \mathrm{kV}$. Nitrogen gas was used as the collision gas (4 psi) and curtain gas (20 psi), whereas zero grade air was used as the nebulizer gas (40 psi) and heated gas (60 psi). Source temperature was set at $600^{\circ} \mathrm{C}$. The collision energy and declustering potential were set at $19 \mathrm{~V}$ and $71 \mathrm{~V}$, respectively. Analyst software (version 1.4, Applied Biosystems) was used to control the LC-MS/MS system, and to acquire and process the data.

\subsubsection{Calibration curve and validation}

Calibration curves for quality control (QC) samples were obtained from the ratio of peak areas of $8-\mathrm{OHdG}$ and $\left[{ }^{15} \mathrm{~N}_{5}\right] 8-\mathrm{OHdG}$ (IS) using $0.1 \mathrm{~mL}$ human urine samples from six humans spiked with $8-\mathrm{OHdG}$ at final concentrations of $0.2,5,10,30,50,100$ nmol/L. Standard curves were also obtained from plotting the peak area ratio against the same six concentrations of the analyte as the spiked urine samples ( $n=6$ for each). The lower limit of quantification (LLOQ) was determined as the lowest standard on the calibration curve that gave a signal-to-noise ratio of more than ten and reached a precision of $20 \%$ and an accuracy of $80-120 \%$. The limit of detection (LOD) was determined as the lowest concentration that gave a signal-to-noise ratio of more than 
three. To evaluate the intra- and inter-day accuracy and precision, the stock solution of 8-OHdG was added to urine at concentrations of 10 and $50 \mathrm{nmol} / \mathrm{L}$ in $0.1 \mathrm{~mL}$ urine. The spiked samples, together with non-spiked samples, were analyzed using HILIC-MS/MS and 8 -OHdG concentrations were calculated using a standard curve. Accuracy was expressed as the ratio of the quantified concentration to the known concentration of 8-OHdG. To evaluate the intra-day precision, the non-spiked samples and the samples spiked at the two levels were prepared five times per day. The inter-day precision was determined using five independent experiments. The precision was calculated as the relative standard deviation (RSD) (\%) of the replicates.

2.4. 8-OHdG analysis by LC-MS/MS using reversed-phase column

On the basis of previously reported LC-MS/MS method [25], chromatographic separation of $8-\mathrm{OHdG}$ and $\left[{ }^{15} \mathrm{~N}_{5}\right] 8-\mathrm{OHdG}$ (IS) in urine samples was performed on a XBridge $\mathrm{C}_{18}$ column $(150 \mathrm{~mm} \times 2.1 \mathrm{~mm}$ i.d., $3.5 \mu \mathrm{m}$, Waters $)$ with a guard column (XBridge $\mathrm{C}_{18}$ column, $10 \mathrm{~mm} \times 10 \mathrm{~mm}$ i.d., $5 \mu \mathrm{m}$, Waters). The elution was run isocratically with a mobile phase consisting of $10 \mathrm{mM}$ ammonium acetate : methanol $(19: 1, \mathrm{v} / \mathrm{v})$ at a flow rate of $0.4 \mathrm{~mL} / \mathrm{min}$. The column temperature was set at $40^{\circ} \mathrm{C}$. The mass spectrometric detection was carried out by the same SRM transitions as the HILIC 
mode.

\section{Results and discussion}

3.1. Mass spectrometry and chromatography

The full scan mass spectrum of $8-\mathrm{OHdG}$ in the positive ESI mode and the fragmentation pattern of protonated molecular ion $[\mathrm{M}+\mathrm{H}]^{+}$observed in this study were consistent with those of previous studies $[17,18,24,26]$. The transition from the molecular ion $[\mathrm{M}+\mathrm{H}]^{+}$to the most intense fragment was recorded in the selective reaction monitoring (SRM) acquisition mode. The main product ions of 8-OHdG and $\left[{ }^{15} \mathrm{~N}_{5}\right] 8-\mathrm{OHdG}$ (IS) were $\mathrm{m} / \mathrm{z} 167.9$ and $173.0[\mathrm{M}+\mathrm{H}-116]^{+}$, respectively. Therefore, the $[\mathrm{M}+\mathrm{H}]^{+} \rightarrow[\mathrm{M}+\mathrm{H}-116]^{+}$transition was used in the SRM mode.

We examined the effect of the acetonitrile concentration in the mobile phase of the HILIC column on the retention time of the analytes. The column was operated under isocratic elution conditions using acetonitrile concentration between 70 to $95 \%$, at the interval of $5 \%$. The retention factor $(k)$ increased with the increasing acetonitrile concentration (Fig. 1). The high content of acetonitrile increases the hydrophilic interactions between the analytes and the stationary phase [33]. At 95\% acetonitrile, 8-OHdG was retained for over 15 min on the column and its peak shape was broad. The 
retention time of charged analytes can be affected by adding salt to the mobile phase due to the electrostatic interactions between the column and analytes [33, 34]. For a salt, we selected ammonium acetate because of its solubility in concentrated acetonitrile solutions, and its volatility at the ion source. The effect of the buffer concentration in the mobile phase on the retention of the analyte was investigated in the range $10-50 \mathrm{mM}$. Increasing the buffer concentration only slightly decreased the retention and did not influence the peak shape (data not shown). Finally, the analytes were separated by isocratic elution with $10 \mathrm{mM}$ ammonium acetate : acetonitrile $(1: 9, \mathrm{v} / \mathrm{v})$. The analyte and the internal standard eluted within 10 min using the optimized mobile phase (Fig. 2, A-1 and A-2).

\subsection{Calibration curve and validation}

The calibration curve for the standard compound was linear $\left(r^{2}>0.999\right)$ for concentrations in the range of $0.2-100 \mathrm{nmol} / \mathrm{L}$ (LLOQ: $0.2 \mathrm{nmol} / \mathrm{L}$ ), which covers the lower range of the reported levels of $8-\mathrm{OHdG}$ in human urine [29], and the slope was $0.00630 \pm 0.00026$ (mean \pm S.D. RSD, $4.1 \%$ ).

Representative SRM chromatograms for the analyte and the internal standard of a urine sample from a non-smoker showed that the physiological components of the urine 
did not interfere with the identification and quantification of the analytes in the chromatograms (Fig. 2, B-1 and B-2). The matrix effect on the mass spectrometric response was evaluated by comparing the slope of the calibration curve with the slope obtained in the presence of urine matrix. Six curves were obtained using six different urine samples, each spiked with six different amounts of 8-OHdG. The mean slope was $0.00655 \pm 0.00043$ (mean \pm S.D., RSD, $6.6 \%$ ), which was almost identical to the mean slope obtained with the standard solutions. This clearly showed that the matrix did not affect the calibration curve. Therefore, $8-\mathrm{OHdG}$ was quantified by using the calibration curve obtained from the standard solution.

The precision and accuracy of $8-\mathrm{OHdG}$ determination in human urine with the HILIC-MS/MS system were examined by adding two different known amounts of 8-OHdG to a urine sample. The RSDs of the intra-day precision assay $(n=5)$ were in the range $2.3-2.6 \%$, and those of the inter-day assay $(n=5)$ were in the range $2.1-4.0 \%$ (Table 1). The accuracy values (\%) of the intra-day study and the inter-day assay were in the range $96-102 \%$. Both intra- and inter-day precision and accuracy values were satisfactory for determining $8-\mathrm{OHdG}$ in human urine.

3.3. Comparison of the HILIC column with a reversed-phase column 
The instrumental detection limit of 8-OHdG measured by the HILIC-MS/MS was 1.0 fmol/injection (signal-to-noise ratio, $\mathrm{S} / \mathrm{N}>3$ ). In contrast, the detection limit achieved with a reversed-phase column and the same detection system was $26.0 \mathrm{fmol} /$ injection under optimal instrumental conditions. Our detection limit of $1.0 \mathrm{fmol}$ was considerably lower than the detection limits reported for other previous LC-MS/MS methods: 20 fmol [17], 7 fmol $(\mathrm{S} / \mathrm{N}=3)$ [18], 5 fmol $(\mathrm{S} / \mathrm{N}=4)$ [19] and $7.5 \mathrm{fmol} /$ injection $(\mathrm{S} / \mathrm{N}=3)$ [27, 31]. The low detection limit of method enables the measurement of basal levels of urinary $8-\mathrm{OHdG}$ in non-exposed healthy subjects that were not quantified in the previous report [27, 31]. Furthermore, the analysis can be completed in $10 \mathrm{~min}$ and doesn't require washing and re-equilibrating the column, which makes it well suited for continuous analyses.

\section{Conclusions}

HILIC-MS/MS provides a selective, sensitive and rapid method for determining 8-OHdG in human urine. The method has acceptable linearity, accuracy, and precision, and is more sensitive than previously described LC-MS/MS methods that have been used in reversed-phase columns. The proposed HILIC-MS/MS method is well suited for large-scale human studies and clinical studies, and would also be applicable to 
analysis of 8-OHdG in not only urine but also other biological fluids such as plasma, serum and saliva, and in tissue.

\section{Acknowledgements}

This study was partly supported by the Industrial Technology Research Grant Program in 2005 from New Energy and Industrial Technology Development Organization (NEDO) of Japan (ID: 05A21705a), by a grant from the Smoking Research Foundation, and by a Grant-in-Aid for Scientific Research from the Ministry of Education, Culture, Sports, Science and Technology, Japan (21790126 and 23406004). 


\section{References}

[1] J.G. Scandalios, Genome Biol. 3 (2002) 1019.1.

[2] J.E. Klaunig, L.M. Kamendulis, Annu. Rev. Pharmacol. Toxicol. 44 (2004) 239.

[3] P. Rossner Jr., V. Svecova, A. Milcova, Z. Lnenickova, I. Solansky, R.J. Sram, Mutat. Res. 642 (2008) 14.

[4] M.S. Cooke, M.D. Evans, M. Dizdaroglu, J. Lunec, FASEB J. 17 (2003) 1195.

[5] M.D. Evans, M. Dizdaroglu, M.S. Cooke, Mutat. Res. 567 (2004) 1.

[6] M.D. Evans, M.S. Cooke, Bioessays 26 (2004) 533.

[7] M.S. Cooke, M.D. Evans, R.M. Burd, K. Patel, A. Barnard, J. Lunec, P.E. Hutchinson, J. Invest. Dermatol. 116(2001) 281.

[8] L.L. Wu, C.-C. Chiou, P.-Y. Chang, J.T. Wu, Clin. Chim. Acta 339 (2004) 1.

[9] K. Sakai, T. Ochi, M. Takeuchi, J. Anal. Bio-Sci. 32 (2009) 297.

[10] G. Guetens, G. De Boeck, M. Highley, A.T. van Oosterom, E.A. de Bruijn, Crit. Rev. Clin. Lab. Sci. 39 (2002) 331.

[11] H.E. Poulsen, S. Loft, H. Prieme, K. Vistisen, J. Lykkesfeldt, K. Nyyssonen, J.T. Salonen, Free Radic. Res. 29 (1998) 565.

[12] K. Shimoi, H. Kasai, N. Yokota, S. Toyokuni, N. Kinae, Cancer Epidemiol. 
Biomarkers Prev. 11 (2002) 767.

[13] J. Cadet, M. Berger, T. Douki, J.-L. Ravanat, Rev. Physiol. Biochem. Pharmacol. $131(1997) 1$.

[14] C. Tagesson, M. Källberg, C. Klintenberg, H. Starkhammar, Eur. J. Cancer 31A (1995) 934.

[15] J.-L. Ravanat, P. Guicherd, Z. Tuce, J. Cadet, Chem. Res. Toxicol. 12 (1999) 802.

[16] I. Holmberg, P. Stål, M. Hamberg, Free Radical Biol. Med. 26 (1999) 129.

[17] J.-L. Ravanat, B. Duretz, A. Guiller, T. Douki, J. Cadet, J. Chromatogr B 715 (1998) 349.

[18] T. Renner, T. Fechner, G. Scherer, J. Chromatogr B 738 (2000) 311.

[19] M.S. Cooke, R. Singh, G.K. Hall, V. Mistry, T.L. Duarte, P.B. Farmer, M.D. Evans, Free Radic. Biol. Med. 41 (2006) 1829.

[20] B. Crow, M. Bishop, K. Kovalcik, D. Norton, J. George, J.A. Bralley, Biomed. Chromatogr. 22 (2008) 394.

[21] B. Malayappan, T.J. Garrett, M. Segal, C. Leeuwenburgh, J. Chromatogr A 1167 (2007) 54.

[22] F. Teichert, R.D. Verschoyle, P. Greaves, J.F. Thorpe, J.K. Mellon, W.P. Steward, P.B. Farmer, A.J. Gescher, R. Singh, Rapid Commun. Mass Spectrom. 23 (2009) 258. 
[23] C.-W. Hu, C.-J. Wang, L.W. Chang, M.-R. Chao, Clin. Chem. 52 (2006) 1381.

[24] L. Sabatini, A. Barbieri, M. Tosi, A. Roda, F.S. Violante, Rapid Commun. Mass Spectrom. 19 (2005) 147.

[25] W.-Y. Hsu, W.T. Chen, W.-D. Lin, F.-J. Tsai, Y. Tsai, C.-T. Lin, W.-Y. Lo, L.-B. Jeng, C.-C. Lai, Clin. Chim. Acta 402 (2009) 31.

[26] A. Weimann, D. Belling, H.E. Poulsen, Nucleic Acids Res. 30 (2002) e7.

[27] M.S. Cooke, R. Olinski, S. Loft, Cancer Epidemiol. Biomarkers Prev. 17 (2008) 3.

[28] M.D. Evans, R. Olinski, S. Loft, M.S. Cooke, FASEB J. 24 (2010) 1249.

[29] A. Pilger, S. Ivancsits, D. Germadnik, H.W. Rüdiger, J. Chromatogr. B 778 (2002) 393.

[30] W. Jian, R.W. Edom, Y. Xu, N. Weng, J. Sep. Sci. 33 (2010) 681.

[31] A. Weimann, D. Belling, H.E. Poulsen, Free Radical Biol. Med. 30 (2001) 757.

[32] E.S. Grumbach, D.M. Wagrowski-Diehl, J.R. Mazzeo, B. Alden, P.C. Iraneta, LC GC N. Am. 22 (2004) 1010.

[33] R.-I. Chirita, C. West, A.-L. Finaru, C.Elfakir, J. Chromatogr. A 1217 (2010) 3091.

[34] S. Drivelos, M.E. Dasenaki, N.S. Thomaidis, Anal. Bioanal. Chem. 397 (2010) 2199. 


\section{Figure Legends}

Fig. 1 Effect of acetonitrile content in the mobile phase on the retention of 8-OHdG.

Fig.2 Representative SRM chromatograms (transition: $\mathrm{m} / \mathrm{z} 284.3 \rightarrow 167.9$ for $8-\mathrm{OHdG}$ and $\mathrm{m} / \mathrm{z} 289.1 \rightarrow 173.0$ for the internal standard $\left.\left(\left[{ }^{15} \mathrm{~N}_{5}\right] 8-\mathrm{OHdG}\right)\right)$ of a standard solution corresponding to $200 \mathrm{fmol} 8-\mathrm{OHdG} /$ injection (A) and a non-smoker urine sample (B) (A-1 and B-1 panels for 8-OHdG; A-2 and B-2 panels for the internal standard). 
Table 1

Hosozumi et al.

Table 1 Precision and accuracy in the determination of 8-OHdG in urine samples

\begin{tabular}{ccccccc}
\hline & \multicolumn{3}{c}{ Intra-day assay $(\mathrm{n}=5)$} & \multicolumn{3}{c}{ Inter-day assay $(\mathrm{n}=5)$} \\
\hline $\begin{array}{c}\text { Added amounts } \\
(\mathrm{pmol} / \mathrm{mL} \text { urine })\end{array}$ & 0 & 10 & 50 & 0 & 10 & 50 \\
$\begin{array}{c}\text { Found } \pm \text { SD } \\
(\mathrm{pmol} / \mathrm{mL} \text { urine })\end{array}$ & $17.5 \pm 0.4$ & $27.0 \pm 0.7$ & $68.5 \pm 1.6$ & $16.8 \pm 0.4$ & $26.9 \pm 0.7$ & $67.5 \pm 2.7$ \\
RSD (\%) & 2.3 & 2.6 & 2.4 & 2.1 & 2.8 & 4.0 \\
Accuracy (\%) & - & 96 & 102 & - & 101 & 101 \\
\hline
\end{tabular}


Figure 1

Hosozumi et al.

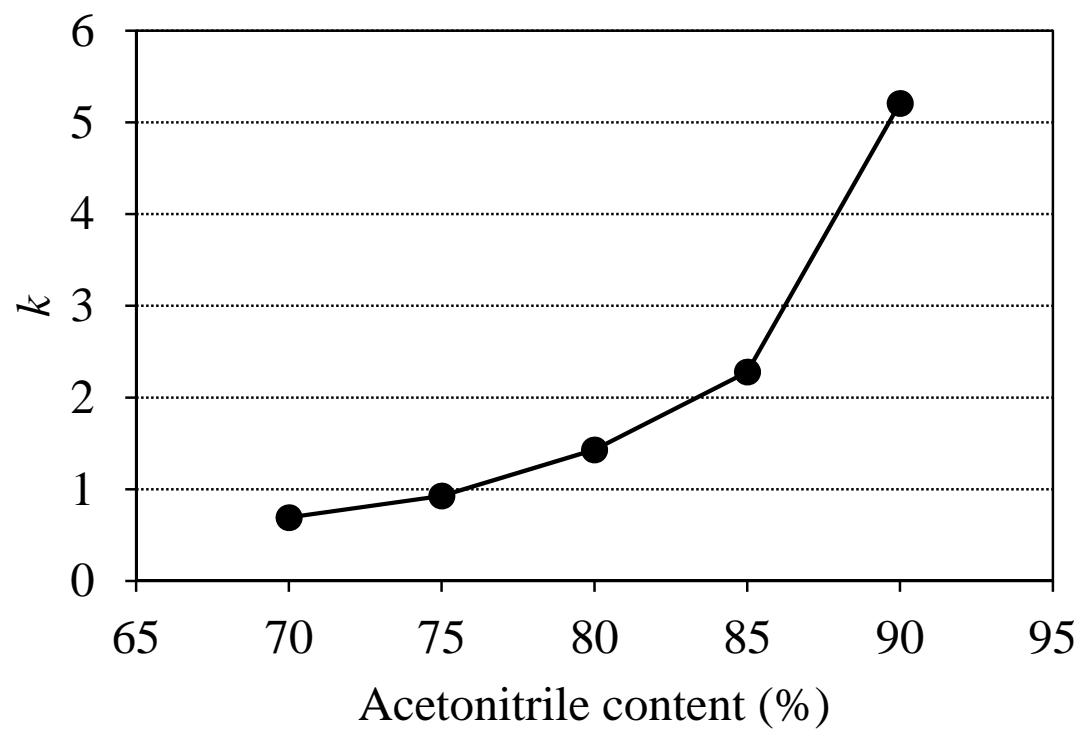


Figure 2

Hosozumi et al.
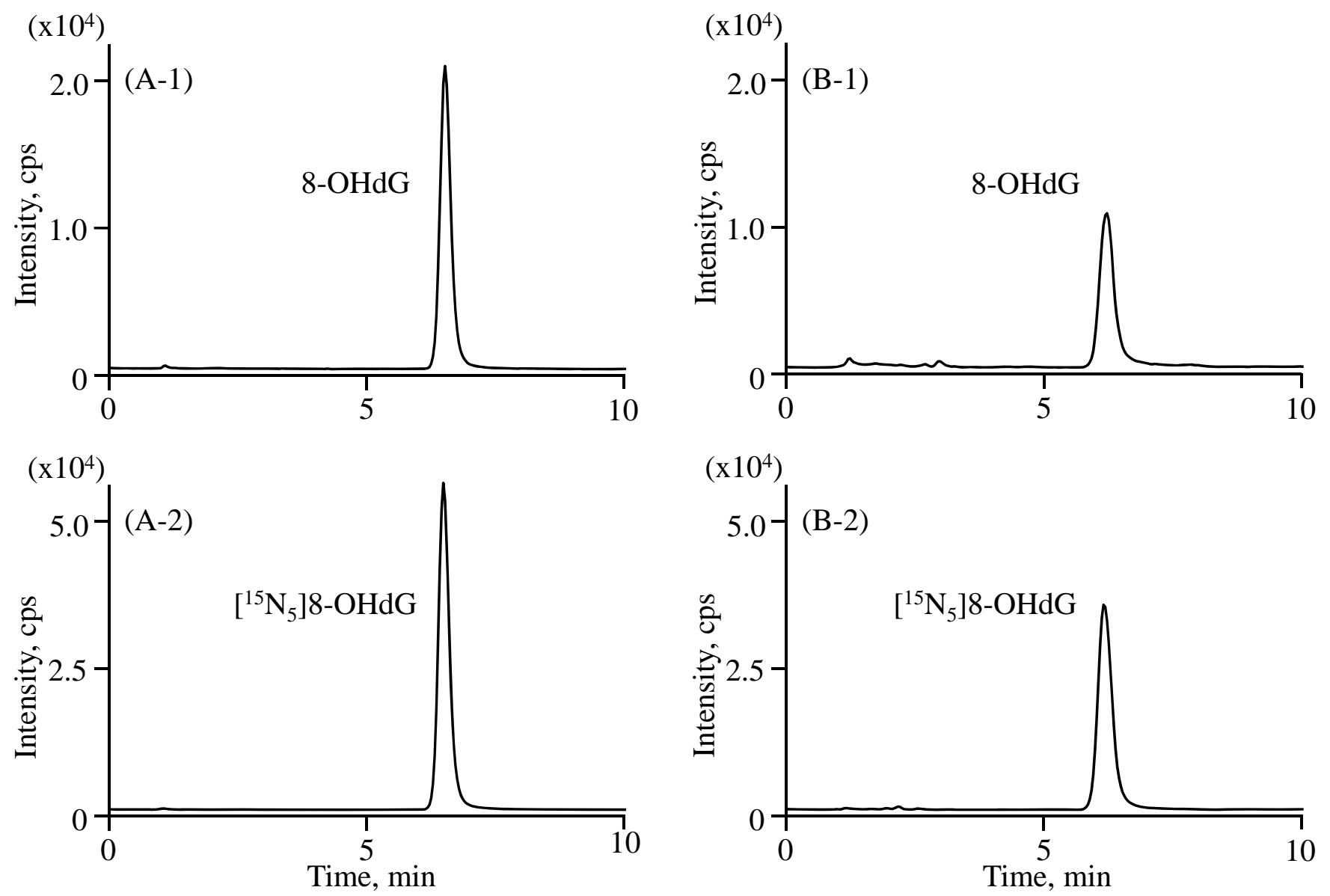